\title{
FORMACIÓN DE PROFESIONALES DE EDUCACIÓN \\ BÁSICA BILINGÜE INTERCULTURAL EN LA SELVA \\ CENTRAL: ALGUNAS PERSPECTIVAS GENERALES \\ DESDE LA CIUDAD DE ATALAYA ${ }^{1}$
}

Pedro P. Soto Canales

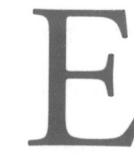

1 presente trabajo — de carácter descriptivo- busca hacer de conocimiento uno de los proyectos en que la Universidad Católica Sedes Sapientiae (UCSS) se ha comprometido a apoyar, nos referimos al Programa de Formación Magisterial Bilingüe Intercultural para beneficio de las comunidades nativas correspondientes a la jurisdicción del Vicariato de San Ramón en la Selva Central. Habiendo concluido el primer ciclo de estudios (Agosto 2007), la intención es presentar de manera general, un acercamiento a la realidad geográfica, la vida académica y tradicional de los jóvenes inmersos en dicho Programa.

\footnotetext{
1 El contenido se basa, especialmente, en la experiencia del autor como uno de los docentes enviados por la universidad para el desarrollo de su asignatura (Historia del Perú I) en coordinación con el Vicariato ya mencionado.
}

$$
-551-
$$




\section{EDUCACIÓN E INTERCULTURALIDAD ${ }^{2}$}

En los sistemas educativos contemporáneos se viene asumiendo como prioritarios el tratamiento de determinados problemas como la violencia, las desigualdades económicas y sociales, la discriminación, el consumismo, el hambre, las migraciones, el racismo, el desarrollo sostenible, las relaciones interculturales, los modelos de comunicación, etc.

En el campo de la educación estos problemas son asumidos como temas que las escuelas pueden incorporar en sus experiencias curriculares debido a que son sumamente importantes para el diseño y construcción del futuro estrechamente relacionado con la práctica de valores. La Educación Intercultural es una alternativa que la educación brinda a la sociedad para el mejoramiento de las relaciones entre los grupos humanos culturalmente distintos; hoy en nuestros días ha demostrado ser uno de los aportes educativos más importantes y significativos del siglo $\mathrm{xx}$ de mayor trascendencia y protagonismo para la educación de todos los países en el siglo XXI debido a su estrecha relación con la construcción de contextos sociales más igualitarios.

En tal sentido, la Educación Intercultural busca disminuir los prejuicios raciales y culturales y los comportamientos discriminatorios en aquellos contextos sociales considerados multiculturales, desarrollando la autoestima, el reconocimiento del otro y optimizando el tratamiento curricular. Teniendo en consideración esto debemos considerar que la Educación Intercultural es un eje transversal o tema transversal y estos son contenidos

2 Máximo Estupiñán, especialista en temas de Interculturalidad y profesor de la UCSS, ha aceptado amablemente entregar estas líneas como introducción para el presente trabajo. Agradecemos, por otro lado, a los profesores Hilario Castilla (Matemáticas y Coordinador Académico-NOPOKI), Darinka Díaz (Lengua y Coordinadora Económica-NOPOKI). 
educativos orientados más allá de lo cognitivo y lo procedimental, están orientados hacia lo actitudinal, dirigidos a desarrollar el pilar del «aprender a convivir». "Aquella educación que permite aprender a todo ser humano el adecuado reconocimiento del otro y de su propia cultura, disminuyendo prejuicios y conductas discriminatorias raciales y culturales, posibilitando el mejoramiento de las relaciones interculturales entre los distintos grupos humanos en contextos sociales multiculturales» (Estupiñán 2007: 33).

En tal medida los objetivos de la Educación Intercultural son básicamente los siguientes:

- Disminuir prejuicios, estereotipos y conductas discriminatorias por motivos raciales y/o culturales.

- Posibilitar el adecuado reconocimiento del otro desarrollando el pleno respeto de las diferencias.

- Reconocer y valorar las diferentes manifestaciones culturales de los diversos pueblos y naciones del mundo y de la propia cultura.

- Optimizar las relaciones interpersonales entre los diversos pueblos y los individuos y las culturas.

Por ello la interculturalidad se traduce socialmente en una convivencia pacífica, en una valoración de las diferencias donde se respetan la totalidad de la dignidad humana y los derechos comunes a todos, en un marco de igualdad, respetando desde las distintas formas de ver, entender y representar el mundo hasta los rasgos físicos, étnicos y culturales, contribuyendo al fortalecimiento de la democracia en una sociedad donde cada cultura o grupo humano participe en mayores condiciones de ciudadanía e igualdad de oportunidades. 


\section{UBicACIÓN GEOGRÁFICA DE LA CIUDAD DE ATALAYA}

Para ubicar geográficamente en dónde está desarrollándose el Programa de Formación Magisterial Bilingüe Intercultural, realizaré dos tipos de descripción. La primera es la ubicación físico-política; la segunda, la demarcación o jurisdicción eclesiástica.

El departamento de Ucayali (del aimara ucayala, que signigica 'ese amigo'), se caracteriza por tener hasta la fecha —año 2007— veintisiete años de existencia, pues mediante Decreto Ley n. ${ }^{\circ} 23099$ del 18 de junio de 1980 es creada en una superficie comprendida de 10241055 kilómetros cuadrados, independizándose así del departamento de Loreto y siendo posteriormente reconocida como Región Ucayali en el año de 1988.

Teniendo como capital la ciudad de Pucallpa (del quechua puka allpa, que significa 'tierra colorada'), el departamento de Ucayali comprende cuatro provincias: Coronel Portillo, Padre Abad, Purus y Atalaya. Estas tres últimas son creadas conjuntamente mediante Ley n. ${ }^{\circ} 23416$ del 1 de junio de $1982 .^{3}$

La provincia de Atalaya es la más extensa del departamento (38924 43 kilómetros cuadrados), teniendo para el año 2005 una población censada de 38104 habitantes, siendo su densidad poblacional de un habitante por kilómetro cuadrado. La provincia de Atalaya se encuentra dividida a su vez en cuatro distritos: Raimondi, Sepahua, Tahuania y Yurua.

En el distrito de Raimondi se encuentra la ciudad capital de la provincia de Atalaya, la cual lleva el mismo nombre (Atalaya), y es donde se desarrolla el Programa de Formación Magisterial Bilingüe Intercultural.

3 La provincia de Coronel Portillo fue anexada al departamento de Ucayali, ya que en sus inicios formaba parte del departamento de Loreto, siendo su fecha de creación el 2 de julio de 1943 , mediante Ley $n .^{\circ} 9815$. 


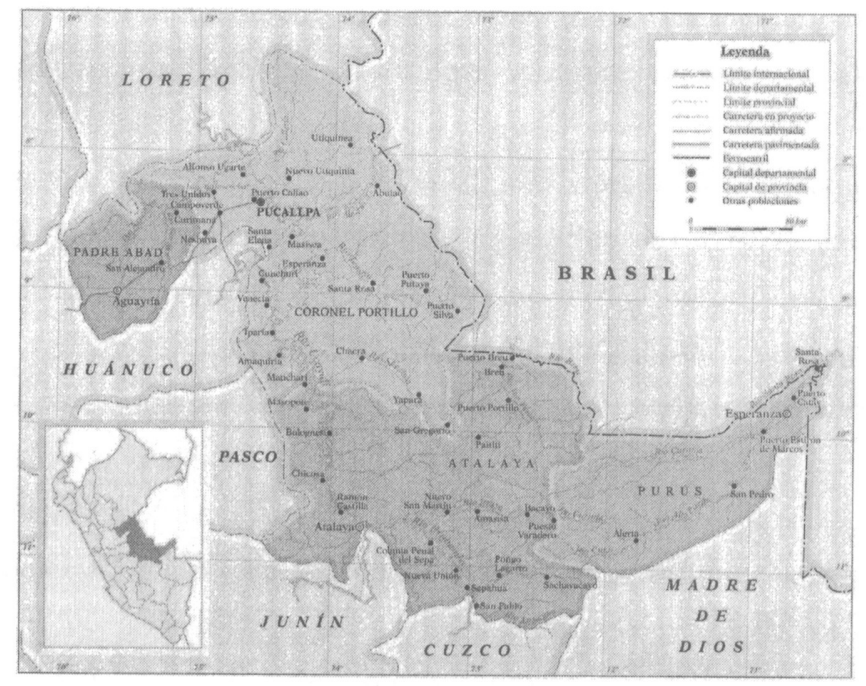

Mapa 1: Departamento de Ucayali 
Desde el siglo XVII, el territorio en estudio ha sido escenario de «misiones» evangelizadoras, especialmente por obra de los hermanos franciscanos. El 5 de febrero de 1900 se crea la Prefectura Apostólica de San Francisco Solano del Ucayali, siendo esta la primera demarcación jurídica eclesiástica realizada el siglo pasado. Posteriormente, en 1925, es elevada a la categoría de Vicariato Apostólico; sobre la naturaleza de una Prefectura o Vicariato, el Derecho Canónico explica que: «el vicariato apostólico o la prefectura apostólica es una determinada porción del pueblo de Dios que, por circunstancias peculiares, ${ }^{4}$ aún no se ha constituido como diócesis, y se encomienda a la atención pastoral de un Vicario apostólico o de un Prefecto apostólico para que las rijan en nombre del Sumo Pontífice» (Sección II: Del pueblo de Dios, Título I, Capítulo I, Can. 307, Inciso 1).

El 2 de marzo de 1956, se da la última división territorial eclesiástica del Vicariato de San Francisco Solano del Ucayali. Mediante la Bula «Cum Petierit» del Papa Pío XII, aparecen tres nuevos Vicariatos: Requena, Pucallpa y San Ramón. Comprendiendo esta última las provincias de Oxapampa (Pasco), Chanchamayo y Satipo (Junín) y, Atalaya (Ucayali).

Actualmente Fray Gerardo Antonio Zerdín Bukovec OFM, cumple la misión de Obispo del Vicariato de San Ramón, tal como fuera nombrado por el papa Juan Pablo II, el 19 de enero del 2002, siendo el cuarto obispo regente que ha tenido dicho Vicariato.

4 El mismo comentario al inciso dice: «El Vicariato Apostólico y, todavía la misma Prefectura Apostólica, responden a la etapa de implantación de la Iglesia en un nuevo territorio. Sus Ordinarios las rigen en nombre del Sumo Pontífice, aunque con derechos análogos a los de un Obispo diocesano». 


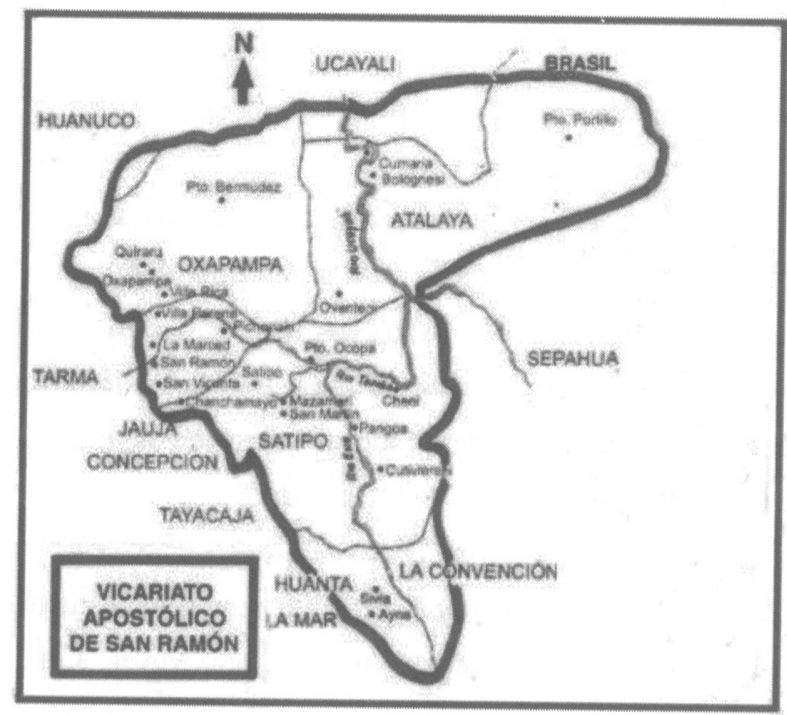

Mapa 2: Vicariato Apostólico de San Ramón 
El Programa de Formación Magisterial Bilingüe Intercultural nació como un proyecto del Vicariato Apostólico de San Ramón, y que actualmente se desarrolla paulatinamente, gracias a la iniciativa de su Obispo, Mons. Gerardo Zerdin OFM. Este programa forma «profesionales en las carreras de Educación Inicial y Primaria, especialmente preparados en los idiomas nativos y para las comunidades del territorio» (Convenio. Art. 3, inciso «a»). Como hice mención en una publicación institucional en el mes de abril, dicho programa va dirigido a «jóvenes provenientes de las diversas comunidades nativas de la zona (Vicariato); no solamente para ser agentes trasmisores de conocimientos, sino también profesionales que atiendan las necesidades de sus comunidades, especialmente en lo que concierne a la preservación de las culturas vivas de las mismas y la promoción de los valores cristianos mediante la educación» (Soto 2007: 6).

El tipo de formación que debe de recibir el futuro educador es tema de profunda preocupación e interés para Mons. Gerardo Zerdin; especialmente de aquel educador que cumplirá su labor en las comunidades nativas bajo su jurisdicción, así nos recuerda que

La escuela que trae valores urbanos expresados en una lengua extraña, representa un quebrantamiento del mundo del niño [...]. Es peor cuando el profesor llega de afuera y desconoce la lengua y cultura nativa. Para él sus alumnos son solo pequeños salvajes de escaso nivel intelectual. En la búsqueda de solución algunos pretenden aislar a los nativos y solo se enseñen la cosmovisión y los valores de las respectivas culturas nativas. Sin embargo, no podemos negar a los nativos el derecho de ampliar su conocimiento del mundo exterior 
[...]. Una propuesta válida es la educación intercultural y bilingüe en la que se parte de la vida concreta y se socializa dentro de su propio medio y con sus propios valores, abriéndose paulatinamente hacia el exterior. El bilingüismo hay que mantener en todas las fases de la educación. (Las cursivas son nuestras. Zerdín 2001: 129)

Para ello, el Vicariato de San Ramón ha solicitado la participación de la Universidad Católica Sedes Sapientiae (UCSS) en la realización de dicho programa, mediante la celebración del «Convenio de Cooperación Institucional» ${ }^{5}$ que tiene como uno de los objetivos en común «formular proyectos, alianzas estratégicas y/o actividades académicas con fines de desarrollo integral de las personas interesadas»(Convenio. Art. 3, inciso «C»).

Por lo tanto, la participación de la UCSS, en dicho programa, es desde el ámbito académico, esto comprende: «Desarrollar [...] módulos académicos presénciales y a distancia [...] con el fin de dar formación a los educandos en la carrera de Educación Inicial y Primaria» (Convenio. Art. 3, inciso «b»). Así también la universidad reconoce y acepta las obligaciones de:

a) Establecer la modalidad de ingreso y propiciar el desarrollo de las carreras de Educación Inicial y Educación Primaria.

b) Facilitar el desarrollo de las asignaturas. Establecer los docentes y administrativos [así] como el material didáctico, monitoreo y la evaluación.

El convenio se celebró el 8 de setiembre del año 2006, en la ciudad de Lima.Firmaron monseñor Gerardo Zerdìn, por parte del Vicariato de San Ramón, y el Gian Battista Bolis, secretario general de la UCSS. 
c) Reglamentar los aspectos de Grados y Títulos. (Convenio. Art.4)

Siendo la universidad una institución que nace y que se desarrolla dentro y hacia la sociedad, su tarea asume tres fines: incrementar los conocimientos (investigar), trasmitir dichos conocimientos (enseñar) y, por último, poderlos aplicar profesionalmente en la sociedad (servicio).

De esta manera la UCSS, unifica los dos primeros fines en el tercero, que es conocido también como de extensión universitaria o Proyección social, esto significa entregar sus servicios fuera de su propia jurisdicción, mediante la formación o capacitación a partir de programas, conferencias, exposiciones, asesorías, etc. Asimismo, el observar siempre que dichos servicios vayan acordes con los programas y currículo que ostenta la universidad. En consecuencia, se concreta una de las funciones últimas de toda institución universitaria — como se encuentra registrado en la Ley del Sistema Universitario Nacional_: «Crear servicios educativos y culturales permanentes de extensión a la comunidad» (Título II, Artículo 7 , inciso «c»).

\section{EL CENTRO DE INVESTIGACIÓN Y FORMACIÓN INTERCULTURAL} «NopoKI»

Para el funcionamiento, tanto del Centro Preuniversitario como para el desarrollo del Programa de Formación Magisterial Bilingüe Intercultural, el Vicariato de San Ramón ha puesto a disposición, las instalaciones del Centro de Investigación y Formación Intercultural Nopoki ('he llegado', en lengua asháninka), ubicado entre el cruce de las calles Quito con Rioja, frente a la plaza Juan Santos Atahualpa, en la ciudad de Atalaya. 


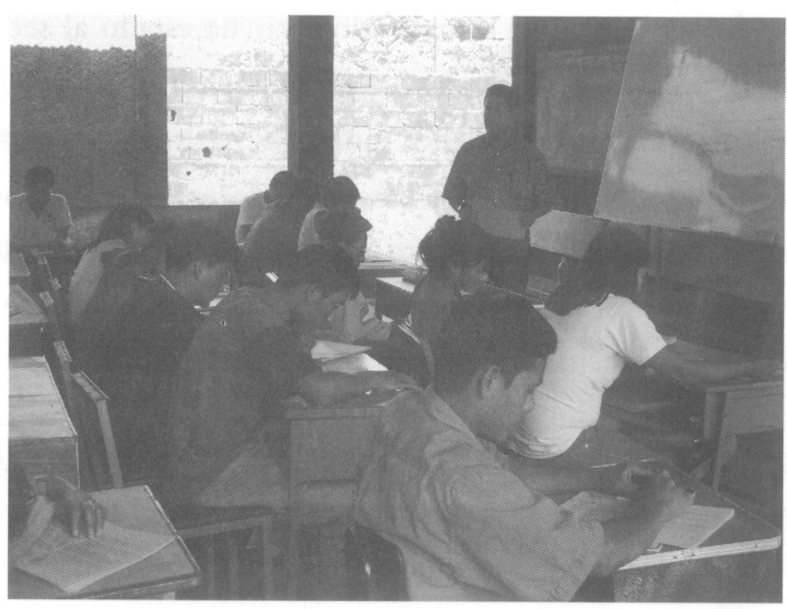

Foto 1: Alumnos del programa, recibiendo una clase de la asignatura de Historia del Perú I, en las instalaciones del centro «Nopoki». 
Los orígenes del centro «Nopoki», se encuentran en las propuestas presentadas por los representantes del Vicariato —entre ellos el padre Gerardo Zerdín quien cumplía el cargo de párroco de Atalaya - ante las autoridades del Ministerio de Educación en el año de 1998, con el fin de crear un centro de formación superior; lamentablemente las acciones realizadas no fueron totalmente favorables, al final y, por otros medios, el Vicariato obtuvo un terreno en donde se construyó el centro en mención.

Desde sus inicios, el centro "Nopoki» ha estado al servicio de la pastoral de la parroquia de Atalaya, especialmente para la formación y reunión de los animadores cristianos indígenas (retiros y jornadas). Aparte de brindar su infraestructura para el desarrollo de las actividades académicas del Programa, actualmente el centro «Nopoki» cumple las funciones de Albergue-internado, especialmente para los jóvenes estudiantes provenientes de las diversas jurisdicciones. Posteriormente será implementado para ser también centro de producción de plantas y animales de consumo, como también la realización de talleres de producción (carpintería, ebanistería, escultura, etc.).

\section{LAS EVALUACIONES DE INGRESO AL PROGRAMA}

Los exámenes para el ingreso a los estudios superiores del Programa de Formación Magisterial Bilingüe Intercultural fueron tomados en las instalaciones del centro «Nopoki», durante los días 15 y 16 de marzo del presente año 2007. Se presentaron setenta postulantes, entre quienes se encontraban jóvenes que habían realizado sus estudios pre-universitarios en el mismo centro Nopoki.

Los postulantes tenían que aprobar dos tipos de evaluación -ambos de carácter eliminatorio-; el primero correspondía al examen escrito de 
conocimientos (jueves 15), elaborado por la UCSS, y el segundo, una entrevista personal (15 y 16 de marzo), donde el jurado estaba conformado por Mons. Gerardo Zerdin y un grupo de docentes nativos de la zona, especialistas en las lenguas de los postulantes, siendo el criterio de evaluación, conocer el nivel de dominio de la lengua nativa.

Las preguntas se agrupaban basándose en tres aspectos del postulante: sobre su persona (formación, familia, aficiones, anécdotas vividas y metas personales); el segundo correspondiente al pueblo de procedencia (descripción, historia y tradición) y, por último, sobre los estudios (interés por la carrera magisterial).

La lista de ingresantes al Programa fue publicado el día viernes 16 de marzo a horas de la tarde, posteriormente se realizó la ceremonia de inauguración del Año Académico, mediante una celebración litúrgica para finalizar en una recepción, en donde participaron los cincuenta y siete nuevos alumnos quienes conforman la primera promoción del Programa.

Cuadro 1: Ingresantes según grupo étnico.

\begin{tabular}{|c|c|c|}
\hline Pueblo / Idioma & Ingresantes & Porcentajes \\
\hline Yine & 06 & $11 \%$ \\
\hline Asháninka & 11 & $19 \%$ \\
\hline Ashéninka & 11 & $19 \%$ \\
\hline Shipibo & 28 & $49 \%$ \\
\hline Nomatsiguenga & 01 & $02 \%$ \\
\hline Total & 57 & $100 \%$ \\
\hline
\end{tabular}


Creo necesario comenzar con definir la malla curricular o currículo universitario como el conjunto de experiencias organizadas por la universidad con el fin de alcanzar los objetivos ya preestablecidos —en este caso- por acuerdo de las instituciones interesadas.

Para ello, la malla curricular para el presente programa contiene las siguientes acciones: el Asesoramiento, las Actividades Planificadas, las Prácticas Profesionales y, sobre todo, las Materias de Estudios ${ }^{6}$ que conforman el «Plan de Asignaturas».

Por lo que he podido experimentar creo pertinente utilizar el término "Tutoría» a cambio de Asesoramiento, ya que no es otra cosa que acompañar al alumno, proporcionándole consejería psicológica, sociológica, espiritual, etc. Es decir, una orientación sistemática para su desarrollo.

Las llamadas Actividades Planeadas se darán paulatinamente buscando responder a aquellas necesidades de los alumnos que no se encuentran presentadas en el plan curricular y/o en las asignaturas. Me refiero a aquellos hábitos básicos y aficiones personales en los estudiantes y que forman parte también de su formación profesional y social.

En los ciclos avanzados, la malla curricular del Programa tiende a propiciar las Prácticas Profesionales, con el fin de facilitar al alumno el dominio práctico de la profesión.

He querido dejar para esta parte el «Plan de Asignaturas»; debo aclarar, ante todo que "las asignaturas [...] son las mismas que se imparten en la sede central de la universidad en Lima, además de complementarse con

6 Muchas veces a una "Asignatura» (tratado o materia que se enseña) se le denomina "Curso", cuando con esta última palabra hacemos referencia al aspecto cronológico, esto es, al tiempo que dura una lección. 
asignaturas y talleres correspondientes a la naturaleza y realidad de la selva central» (Soto 2007: 6).

Durante el primer ciclo, las asignaturas han estado orientadas al área de Estudios Generales. En una primera etapa se han impartido las asignaturas desarrolladas por los docentes enviados por la Universidad —-según convenio_- y en una segunda etapa, las asignaturas desarrolladas por los docentes nativos del Centro de Investigación y Formación Intercultural Nopoki.

Cuadro 2: Asignaturas del ciclo 2007-I

\begin{tabular}{|c|c|c|}
\hline Asignatura & Profesor & Institución \\
\hline Historia del Perú I & Pedro P. Soto Canales & UCSS \\
\hline $\begin{array}{c}\text { Antropología religiosa } \\
\text { Metodología de los Est. } \\
\text { Superiores }\end{array}$ & Edgar Acuña & UCSS \\
\hline Matemática Básica & Hilario Castilla Cruz & NOPOKI \\
\hline Lengua Castellana & Darinka Pacaya Díaz & NOPOKI \\
\hline Idioma Shipibo & Saúl Escobar Rodríguez & NOPOKI \\
\hline Idioma Asháninka & Esaú Zumaeta & NOPOKI \\
\hline Idioma Yine & Remigio Zapata. & NOPOKI \\
\hline
\end{tabular}

El trabajo académico de las asignaturas correspondientes al convenio ha tenido dos modalidades de desarrollarse. La primera, presencial (marzoabril), corresponde al desarrollo de las mismas por un tiempo de quince días, con un intenso régimen de trabajo intenso (véase cuadro 3). La segunda, a distancia, corresponde a los meses siguientes (mayo-julio), donde los alumnos 
han enviado — de manera mensual ${ }^{7}$ sus trabajos de investigación a la sede principal de la UCSS, en Lima. Después de ser calificados y con sus respectivas observaciones fueron devueltos al centro Nopoki, en la ciudad de Atalaya, para ser retomados por los asesores o tutores del programa hasta el regreso de los docentes de la UCSS, que tomaron los exámenes finales en el mes de agosto.

Cuadro 3: Horario de estudios de las asignaturas generales (convenio)

\begin{tabular}{|c|c|c|c|c|c|}
\hline Horario/Día & Lunes & Martes & Miércoles & Jueves & Viernes \\
\hline $8: 00-9: 30$ & $\begin{array}{c}\text { Historia del } \\
\text { Perú I }\end{array}$ & $\begin{array}{c}\text { Antropología } \\
\text { Religiosa }\end{array}$ & $\begin{array}{c}\text { Historia del } \\
\text { Perú I }\end{array}$ & $\begin{array}{c}\text { Antropología } \\
\text { Religiosa }\end{array}$ & $\begin{array}{c}\text { Metodología } \\
\text { de Estudios } \\
\text { Sup. }\end{array}$ \\
\hline $9: 45-11: 15$ & $\begin{array}{l}\text { Metodología } \\
\text { de Estudios } \\
\text { Sup. }\end{array}$ & $\begin{array}{c}\text { Historia del } \\
\text { Perú I }\end{array}$ & $\begin{array}{c}\text { Antropología } \\
\text { Religiosa }\end{array}$ & $\begin{array}{c}\text { Metodología } \\
\text { de Estudios } \\
\text { Sup. }\end{array}$ & $\begin{array}{l}\text { Historia del } \\
\text { Perú I }\end{array}$ \\
\hline $11: 30-13: 00$ & $\begin{array}{c}\text { Antropología } \\
\text { Religiosa }\end{array}$ & $\begin{array}{c}\text { Metodología } \\
\text { de Estudios } \\
\text { Sup. }\end{array}$ & $\begin{array}{l}\text { Metodología } \\
\text { de Estudios } \\
\text { Sup. }\end{array}$ & $\begin{array}{c}\text { Historia del } \\
\text { Perú I }\end{array}$ & $\begin{array}{c}\text { Antropología } \\
\text { Religiosa }\end{array}$ \\
\hline \multicolumn{6}{|c|}{ DESCANSO } \\
\hline $15: 00-16: 00$ & $\begin{array}{l}\text { Historia del } \\
\text { Perú I }\end{array}$ & $\begin{array}{c}\text { Antropología } \\
\text { Religiosa }\end{array}$ & $\begin{array}{c}\text { Historia del } \\
\text { Perú I }\end{array}$ & $\begin{array}{c}\text { Antropología } \\
\text { Religiosa }\end{array}$ & $\begin{array}{c}\text { Metodología } \\
\text { de Estudios } \\
\text { Sup. }\end{array}$ \\
\hline $16: 00-17: 00$ & $\begin{array}{l}\text { Metodología } \\
\text { de Estudios } \\
\text { Sup. }\end{array}$ & $\begin{array}{c}\text { Historia del } \\
\text { Perú I }\end{array}$ & $\begin{array}{c}\text { Antropología } \\
\text { Religiosa }\end{array}$ & $\begin{array}{l}\text { Metodología } \\
\text { de Estudios } \\
\text { Sup. }\end{array}$ & $\begin{array}{c}\text { Historia del } \\
\text { Perú I }\end{array}$ \\
\hline $17: 00-18: 00$ & $\begin{array}{c}\text { Antropología } \\
\text { Religiosa }\end{array}$ & $\begin{array}{l}\text { Metodología } \\
\text { de Estudios } \\
\text { Sup. }\end{array}$ & $\begin{array}{c}\text { Metodología } \\
\text { de Estudios } \\
\text { Sup. }\end{array}$ & $\begin{array}{l}\text { Historia del } \\
\text { Perú I }\end{array}$ & $\begin{array}{c}\text { Antropología } \\
\text { Religiosa }\end{array}$ \\
\hline
\end{tabular}

7 Los trabajos tenían que ser redactados tanto en castellano como en el idioma nativo del alumno. Esta modalidad se dio con el fin de apoyar la labor de los asesores y docentes residentes en Nopoki (Atalaya).

$$
-566-
$$




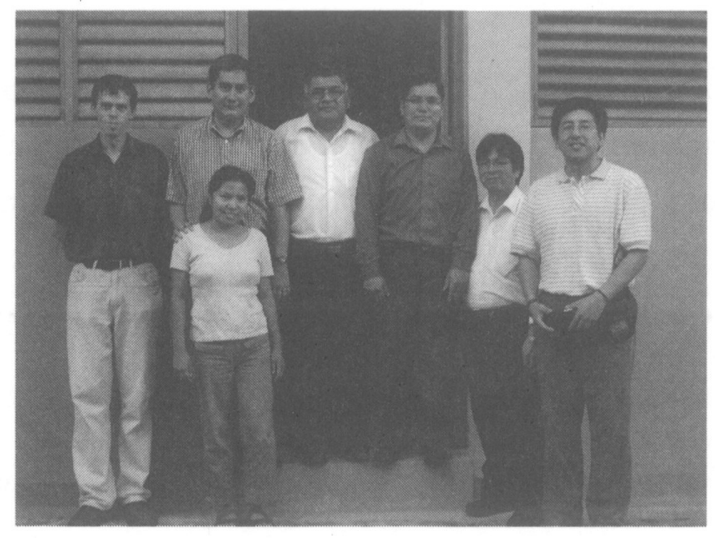

Foto 2: Grupo de profesores: De izquierda a derecha: P. Juan Miedzianowski, Darinka Pacaya Díaz (adelante), Pedro Soto Canales, Hilario Castilla Cruz, Edgar Acuña, Saúl Escobar Rodríguez y Giovanni Sandoval. 
En conclusión, el primer ciclo correspondiente al Programa de Formación Magisterial Bilingüe Intercultural, ha estado conformado por ocho asignaturas generales, entre las cuales cabe destacar la presencia de asignaturas correspondientes a la realidad inmediata del alumno, nos referimos al perfeccionamiento en la lengua nativa de los participantes del Programa.

\section{LOS ALUMNOS DEL PROGRAMA}

Al finalizar el primer ciclo académico en el mes de agosto, el número de alumnos participantes del Programa de Formación Magisterial Bilingüe Intercultural, corresponde a 52. Se percibe la diferencia con el número de ingresantes en el mes de marzo (véase cuadro 1).

A partir de los documentos de matricula, podemos ubicar el origen étnico de los 52 alumnos.

Cuadro 4: Alumnos al finalizar el primer ciclo, según grupo étnico

\begin{tabular}{|l|c|c|c|c|}
\hline \multirow{2}{*}{ Grupo étnico } & \multicolumn{2}{|c|}{ Sexo } & \multirow{2}{*}{ Total } & \multirow{2}{*}{ Porcentaje } \\
\cline { 2 - 3 } & Femenino & Masculino & & \\
\hline Yine & 03 & 02 & 05 & $10 \%$ \\
\hline Asháninka & 02 & 07 & 09 & $17 \%$ \\
\hline Ashéninka & 05 & 06 & 11 & $21 \%$ \\
\hline Shipibo & 04 & 23 & 27 & $52 \%$ \\
\hline Total & 14 & 38 & 52 & $100 \%$ \\
\hline
\end{tabular}

Los jóvenes de origen Yine, proceden de las comunidades nativas como son Pocani y Ramón Castilla. Conocidos también como Piro, 
sus poblaciones viven en el curso bajo del río Urubamba, en los ríos del departamento de Madre de Dios, inclusive existen comunidades yine en territorio brasileño.

Por otra parte, los jóvenes Asháninkas tienen dos puntos centrales de referencia; en primer lugar, se encuentran las localidades del Puerto Ocopa, cuenca del río Tambo, el distrito de Mazamari, todas correspondientes a la provincia de Satipo, departamento de Junín. En segundo lugar, los que proceden del distrito de Raimondi en la misma provincia de Atalaya, departamento de Ucayali.

Unidos a los asháninkas, se encuentran los ashéninkas, quienes proceden - la gran mayoría - de las localidades de Oventini (Atalaya) y de las riberas del río Ucayali, todos correspondientes al distrito de Raimondi, en la provincia de Atalaya, departamento de Ucayali. Cabe resaltar que los ashéninkas, asháninkas, asháninga, atiri, entre otros, son diversos grupos étnicos que se diferencian por motivos dialécticos y sociales, pero todos tienen en común salir de un solo tronco que es el Asháninka.

Por último, tenemos a los jóvenes shipibos con doble procedencia, los de la ciudad de Pucallpa, que se encuentra en la provincia de Coronel Portillo, y es capital del departamento de Ucayali, y los que proceden de las comunidades que se encuentran en los alrededores del río Ucayali, en el distrito de Tahuania en la provincia de Atalaya, departamento de Ucayali.

Como se puede observar, la riqueza de la Amazonia peruana es amplia, no solamente en lo que se refiere a las culturas que la conforman, sino a los pueblos que la integran y, con ellos, a las familias lingüísticas que los unen. $\mathrm{Al}$ respecto, no hay una respuesta totalmente científica concerniente 
a la cantidad de familias lingüísticas existentes en la región amazónica, ${ }^{8}$ es así como desde el punto de vista de la Arqueología se propone decir que «las etnias de la selva peruana se cuentan por decenas y hablan lenguas propias. Se consideran que son más de treinta los idiomas vigentes, aunque varios de ellos tiene raíces comunes por estar emparentados» (Kauffmann 2002: 661).

Cuadro 5: Familias Lingüísticas correspondientes a los alumnos del Programa9

\begin{tabular}{|c|c|}
\hline Familia Lingüística & Grupos Etnicos \\
\hline $\begin{array}{l}\text { Arahuaca } \\
\text { (Arawacs?) }\end{array}$ & $\begin{array}{l}\text { Amuesha (yanesha) } \\
\text { Asháninka } \\
\text { Culina (madija) } \\
\text { Chamicuro } \\
\text { Mashiguenga } \\
\text { Nomatsiguenga } \\
\text { Piro (yine). }\end{array}$ \\
\hline Pano & $\begin{array}{l}\text { Amahuacas } \quad \text { (yora) } \\
\text { Capanahuas (nuquencaibo) } \\
\text { Cashibo-catacaibos (uni) } \\
\text { Cashinahuas (junikuin) } \\
\text { Mayorunas (matse) } \\
\text { Nahuas } \\
\text { Sharanahuas } \\
\text { Shipibos-conibos } \\
\text { Yaminahuas }\end{array}$ \\
\hline
\end{tabular}

8 Algunos estudiosos hacen referencia a 17 familias lingüísticas, mientras que otros fundamentan que son 16, inclusive se propone 15 familias lingüísticas. Entre estas tenemos Arahua, Arahuaca, Bora, Cahuapana, Candoshi, Harakmbut, Huitoto, Jibaro, Pano, Peba-yagua, Simaco, Tacana, Ticuna, Tucano, Tupí-guaraní y Zápar, entre otras. Esto sin contar las 30 lenguas más con sus diversos dialectos.

9 Se escriben con negrita y cursiva los grupos étnicos de donde proceden los alumnos. 


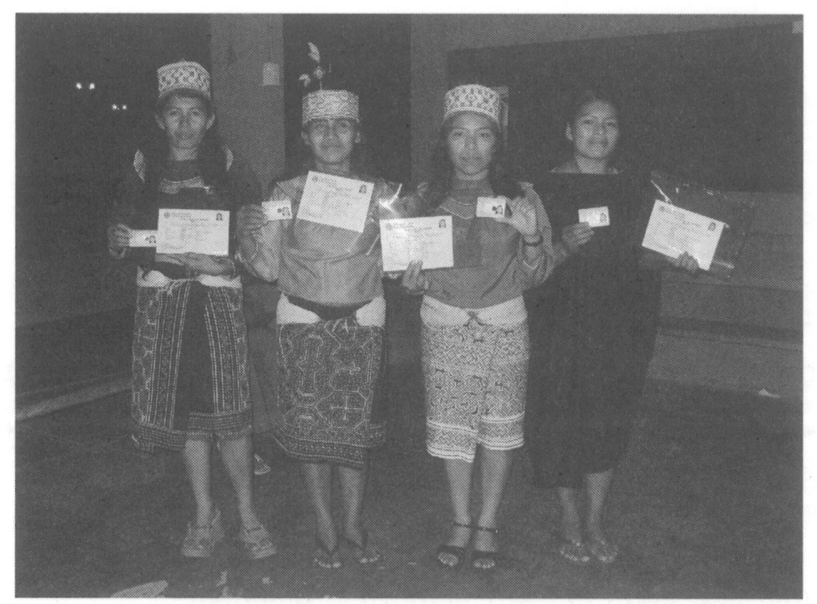

Foto 3: Alumnas del Programa.

De izquierda a derecha: Ángela Sánchez (shipibo), Dayli Linares (shipibo), Jovita Vásquez (shipibo) e Irma Torres (ashéninka) 


\section{EL «CHAMANISMO»: LA TRANSMISIÓN \\ DE CULTURA A PARTIR DE LOS RITOS}

\section{Entrevista al alumno Arturo Valles Izquierdo ${ }^{10}$}

\section{¿Desde cuándo practicas el Chamanismo?}

La práctica y aprendizaje del chamanismo se puede dar a partir de los 10 años de edad. En mi caso comencé a ejercerlo desde los 19 años, actualmente tengo 26 años de edad.

\section{¿Es hereditario, es decir, tu padre también es Chamán?}

Mi padre se llama Víctor Izquierdo Ruiz, tiene actualmente 65 años de edad, y es maestro chamán, también lo fue mi abuelo — su padre— Lino Valles Ruiż, quien falleció a los 75 años de edad, en el año 2006.

Yo participaba en las ceremonias de ayahuasca de mi padre, participaba tomando para ver visiones —es que los chamanes ven por medio de visiones lo que piensas - mi padre veía que yo tenía vocación de chamán para ser su reemplazante. No es necesario u obligatorio seguir una tradición familiar, es decir, que el hijo sea maestro chamán como el padre.

Mi padre me entregó las fuerzas y las canciones, esto significa que las «fuerzas» son el «don» de sanidad y de buenas visiones.

10 Oriundo de la comunidad nativa "9 de octubre», perteneciente a la jurisdicción del distrito de Tahuania, provincia de Atalaya, departamento de Ucayali.

$$
-572-
$$




\section{¿Qué significa ser Chamán?}

Significa tener buena visión, saber sanar y purificar; también existe mala visión para hacer daño o brujería.

En la lengua shipiba, el chamán es un Onaya que quiere decir sabio, capaz de sanar enfermos, y al brujo se le llama Yobe, es la persona mala que también vive dentro de una comunidad nativa. ${ }^{11}$

\section{¿Qué es lo primero que debe aprender un futuro Chamán?}

Para aprender ser un chamán, se debe realizar una «dieta» de aproximadamente seis meses $y$, posteriormente, veinte días de ayuno. Es un tiempo propicio para recibir fuerzas, mediante oraciones hacia el «rey del universo» ${ }^{12}$ que es Dios, el dios católico.

Algunos hacen hasta un año de dieta y ayuno; yo solamente he hecho el primero, el de seis meses, en donde hacía tres oraciones espontáneas al día, dirigidas hacia Dios para que me entregue ese «don» de curar, muchas veces se reza también el Padre Nuestro.

\section{¿Cómo es una Ceremonia de Curación?}

La casa de curación debe ser la casa de uno de los chamanes, durante la ceremonia ellos se sientan por fila, tres chamanes y sus alumnos. El chamán encargado debe hacer que la ceremonia sea impresionante; las ceremonias en mi comunidad se realizan especialmente entres las 8:30 p. m. hasta las 2:00 a. m.

11 El término "chamán» procede del Asia Central y de Siberia, tiene su origen en el grupo lingüístico tungús, donde se escribe utilizando la «s», pasó a la lengua española — por medio del francés - escribiéndose con "c», como lo propone la Real Academia Española(RAE).

12 Resalta en la vida cotidiana de estos hombres de la selva, toda una cultura mítica en relación a la luna en donde todo es mágico y pan naturalista. 
Se utiliza en la ceremonia el «cachimbo», ${ }^{13}$ tabaco, agua florida, el ayabuasca ${ }^{14}$ que se mezcla con el chacuruma ${ }^{15}$ —estos últimos se preparan en la cocina hirviéndolos por cuatro horas aproximadamente. Posteriormente se hace el «acarado», previo a la ceremonia.

Se comienza realizando una oración al dios del universo, también se pide a los hermanos incas para que sean intermediarios, pues nos consideramos descendientes de ellos. Después de haber tomado la ayahuasca - media hora antes - los participantes comienzan a tener mareos, esto es la fuerza de la ayahuasca, el chamán comienza a sentirse levantado sobre la tierra. Y comienza a ver lo trascendente de las personas, se realizan cantos según las necesidades o la situación del paciente. Una mesada puede realizarse por diversas razones, como por ejemplo, para el buen viaje de los amigos. Como para ustedes yo les cantaría:

\author{
Westiora nete bari pikotaiki \\ Kikin penebires jatibien \\ Jato oini chosko mai pekao \\ Jato oini benvobo y tan aybovo \\ Wetsako ibano jakonash kai \\ Ixon jaton shobonko kama
}

\section{Un día el sol ha salido muy \\ brilloso para alumbrar}

A los cuatro extremos de la

Tierra para que los hombres

$\mathrm{Y}$ las mujeres que viajan

A otro lugar y que vayan

muy bien hasta llegar a su casa.

13 Especie de pipa.

14 Palabra de origen quechua: aya waska que significa 'soga del muerto'; es una liana propia de la llanura amazónica, que, mezclada con otras hierbas, produce una bebida de poder hipnótico.

15 Planta medicinal. 
El chamán sigue cantando a las personas hasta que deje de tener las visiones ocasionadas por el ayahuasca —aproximadamente entre cuatro a cinco horas-; no es obligatorio que tomen ayahuasca los pacientes. Al siguiente día, se hace dieta hasta la el mediodía.

\section{¿Puedes resumir todo lo anterior en pocas palabras?}

El chamán da a los pacientes la bienvenida, becanwe, que significa 'vengan'. Después hace las peticiones al padre del universo, para que le dé fuerzas. Se invita a los participantes a que se abandonen al poder de curación del chamán. ${ }^{16}$

El chamán comienza a orar e inhalar el cachimbo y usa el agua florida, pasando y soplando por las manos, la cabeza y todo el cuerpo para dar un diagnóstico. De allí, el chamán chupa con los labios la parte infectada de donde sale el daño o solamente con canciones - yo he sacado una espina de pescado- de ahí todo sale, cual fue el daño y quién se lo hizo. ${ }^{17}$

¿Cuánto se cobra por la realización de una ceremonia?

En mi comunidad, por curaciones el precio es entre 50, 30 y hasta 20 soles; en cambio, en la ciudad el precio es de 50 soles. Pero si es para unir parejas el precio es otro. En la comunidad es 200 ó 300 soles, mientras que en la ciudad sería 100 soles.

16 Muchos estudios promueven reconocer al Chamán como el practicante de una medicina tradicional, la cual se encuentra compuesta por toda una farmacología indígena o medicina folclórica basada en las enseñanzas empíricas de los antiguos o ancestros de las comunidades nativas (transmisión de conocimientos o cultura para la curación de enfermedades).

17 He encontrado una referencia al mismo acto ritual en un artículo del padre Secundino García, OP. Realizado en 1937, pero entre los brujos de la etnia de los «Machiguengas». 


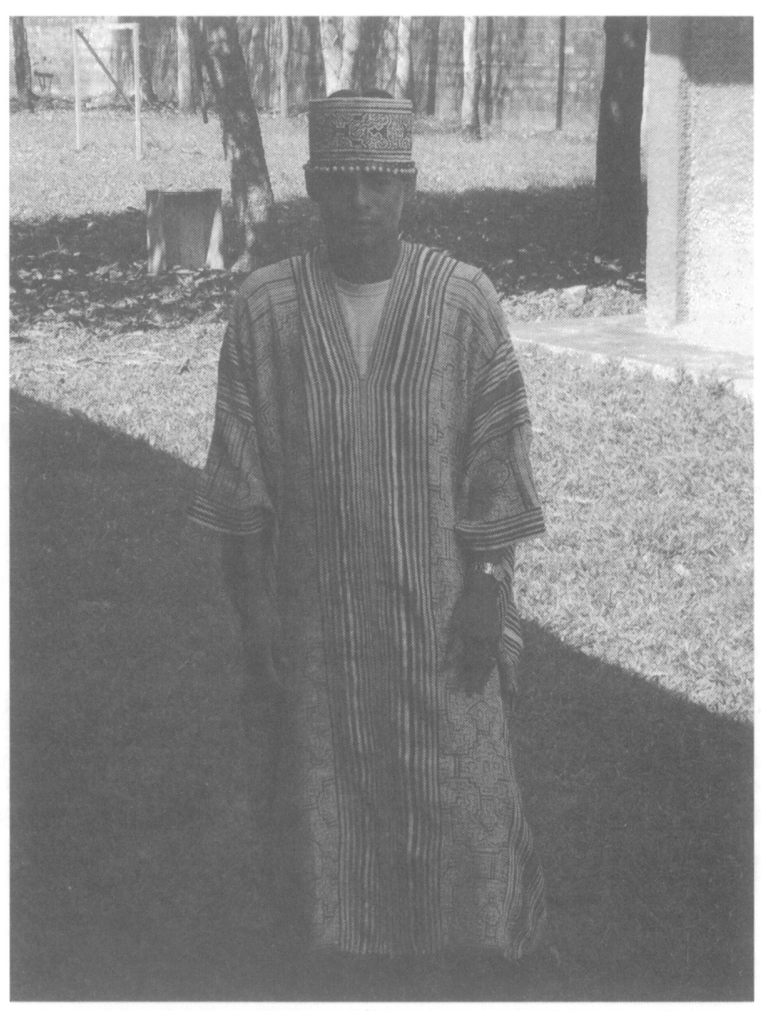

Foto 4: Alumno Arturo Valles Izquierdo. 


\section{BIBLIOGRAFÍA}

ECHEVARRíA, Lamberto de (dir.)

1988 Código de Derecho Canónico. Edición Bilingüe y Comentada. Madrid: BAC.

Estupiñán Maldonado, Máximo

2007 Educación e Interculturalidad: Caracteristicas, fundamentos y componentes. Lima: UCSS.

García Cáceres, Uriel

2006 Enciclopedia Temática del Perú. Tomo I y II: Salud. Lima: El Comercio S.A.

Kauffmann Doig, Federico

2002 Historia y Arte del Perú Antiguo. Tomo IV. Lima: PEISA.

Luca Di Paolo, Angela de

1994 "Presentación. En Arévalo Valera, Guillermo. Medicina Indígena: Las plantas medicinales y su beneficio en la salud. Lima: Aidesep, pp.13-15.

Macerlean, A.

1999 «Ucayali». En Enciclopedia Católica. <www.enciclopediacatolica.com/u/ ucayali.htm>. Consulta hecha en 21/8/2007.

Silva Santisteban, Fernando

2005 El Primate Responsable: Antropobiología de la conducta. Lima: Fondo Editorial del Congreso del Perú. 
Soto Canales, Pedro P.

2007 «Educación bilingüe intercultural en Atalaya». Sedes, Quincenario Informativo, аก๊о VII, n. ${ }^{\circ} 66$. UCSS, p. 6.

Tauro del Pino, Alberto

2001 Enciclopedia Ilustrada del Perú. Tomos I - XVII. Lima: Peisa.

AA. VV.

2006 La vida del pueblo Matsiguenga: Aporte etnográfico de los Misioneros Dominicos al estudio de la Cultura Matsiguenga (1923-1978). 1.a ed. Lima: Centro Cultural José Pío Aza.

Vicariato de San Ramón

s.f. "Historia y Actividades de las Misiones Franciscanas en la Amazonia peruana». En Vicariato de San Ramón. <www3.planalfa.es/ misionesfranciscanas/vicariato_de_san_ramon.htm>. Consulta hecha en 21/8/2007.

Zerdín BuKovec, Gerardo Antonio

2001 «Interculturalidad». En Actas del I Congreso de Educación Religiosa Champagnat. Lima: UPMCH, pp. 125-132. 